Document downloaded from:

http://hdl.handle.net/10251/70555

This paper must be cited as:

Acelas Delgado, P.; Arce Vila, P.; Guerri Cebollada, JC.; Castellanos Hernández, WE. (2012). Evaluation of the MDC and FEC over the quality of service and quality of experience for video distribution in ad hoc networks. Multimedia Tools and Applications. 1-21. doi:10.1007/s11042-012-1111-3.

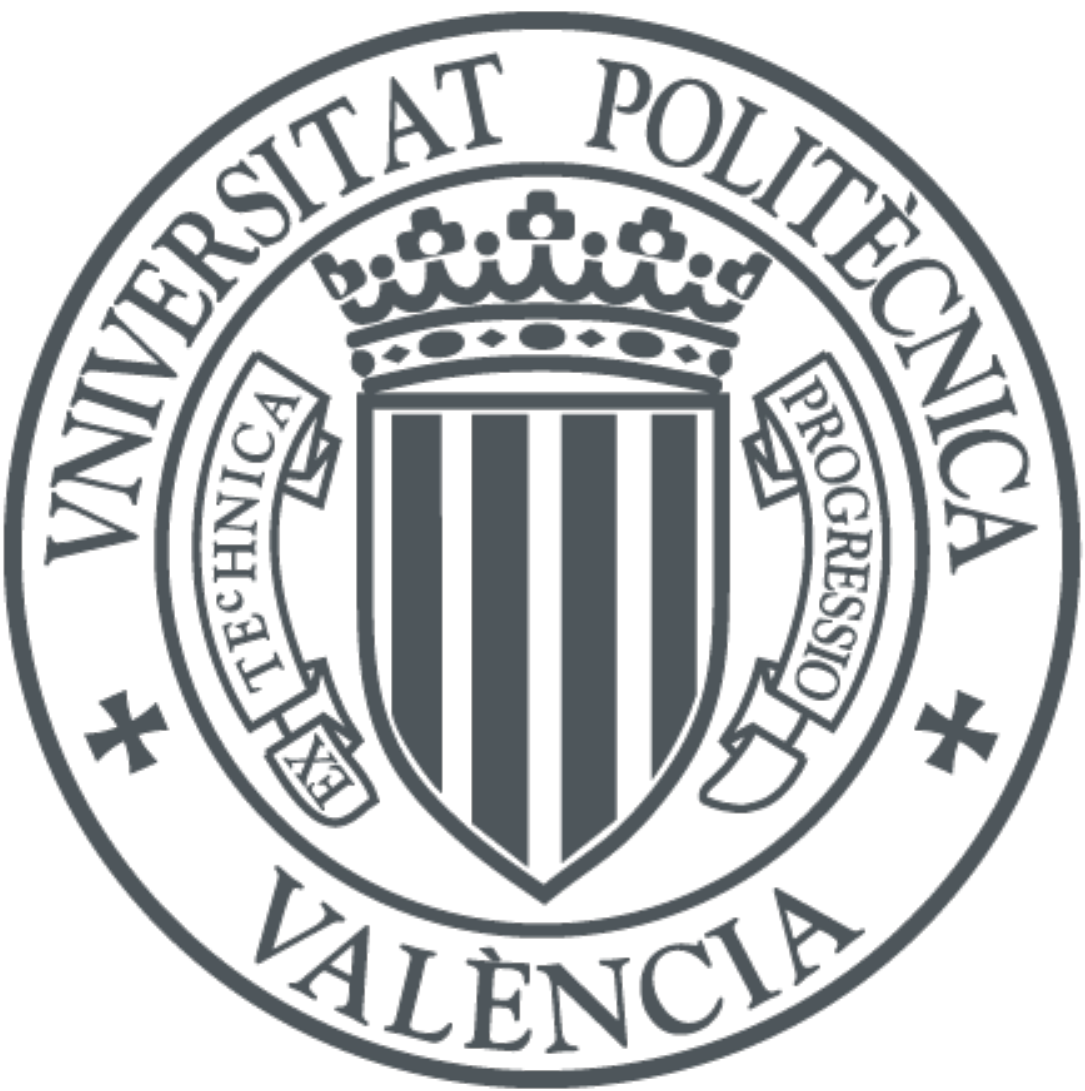

The final publication is available at

http://dx.doi.org/10.1007/s11042-012-1111-3

Copyright Springer Verlag (Germany)

Additional Information 


\title{
Evaluation of the MDC and FEC over the Quality of Service and Quality of Experience for Video Distribution in Ad Hoc Networks
}

\author{
P. Acelas, P. Arce, J. C. Guerri , W. Castellanos \\ Multimedia Communication Group \\ ITEAM Institute, Universitat Politècnica de València, 46022 Valencia, Spain \\ patacdel@iteam.upv.es, paarvi@iteam.upv.es,jcguerri@dcom.upv.es,wilcashe@upv.es
}

Tel: 963879717 , Fax: 963879583

\begin{abstract}
Mobile ad hoc networks (MANETs) offer an excellent scenario for deploying communication applications because of the connectivity and versatility of this kind of networks. In contrast, the topology is usually extremely dynamic causing high rate of packet loss, so that ensuring a specific Quality of Service (QoS) for real-time video services becomes a hard challenge. In this paper, we evaluate the effect of using Multiple Description Coding (MDC) and Forward Error Correction (FEC) techniques for improving video quality in a multimedia content distribution system. A hybrid architecture using fixed and wireless ad hoc networks is proposed, which enables the use of multipoint-to-point transmission. MDC and FEC mechanisms can be combined with multipath transmission to increase the network efficiency and recover lost packets, improving the overall Quality of Experience (QoE) of the receiver. Simulations have been analyzed paying attention to objective parameters (Peak Signal to Noise Ratio, Packet Delivery Ratio, Decodable Frame Rate and interruptions) and subjective parameters. Results show that MDC increases the probability of packet delivery and FEC is able to recover lost frames and reduce video interruptions in moderate mobility scenarios, resulting in the improvement of video quality and the final user experience.
\end{abstract}

Keywords. Ad Hoc Networks, Video Streaming, Multiple Description Coding, Forward Error Correction, Quality of Service, Quality of Experience

\section{INTRODUCTION}

The convergence of different advances in wireless technology and mobile devices lets ad hoc networks become again an attractive subject of study in many areas (emergency services, social networks, home networks, etc.). The evolution of wireless networks towards the next generation $4 \mathrm{G}$ technologies includes different medium access (both cellular and 802.11 networks) and protocols (based on IP stack), and involves ad hoc networks as part of that architecture. Moreover, the evolution of mobile devices has led to an increment of their CPU performance, storage capacity, bandwidth and energy management. In the near future, these constant improvements will allow such devices to efficiently carry out the possible tasks of a node in an ad hoc network: server, client and router.

On the other hand, users expect to access over ad hoc networks to the same kind of services currently accessed through infrastructured wireless networks (3G and WLAN), such as video streaming services (e.g. Youtube) or online music services (e.g. Spotify). These real-time services require that network offers Quality of Service (QoS) by ensuring sufficient bandwidth and an upper bound in delay, jitter and loss rate. Furthermore, the Quality of Experience (QoE) perceived by the user should be measured in order to affirm the usefulness of the service.

However, it is well known that streaming videos across Mobile Ad hoc Networks (MANETs) introduces a lot of challenges. The own definition of MANET, which entails wireless links, multi hop routes and mobility of nodes, is the main reason of the biggest problems: a considerable reduction of the available bandwidth and a dynamic topology with frequent route breakages [1]. All these cause packet loss and negative effects in traffic throughput.

Different solutions have been proposed to overcome these challenges in MANET networks, some of them based on cross-layer mechanisms, enhancements to MAC layer or new routing protocols designed to consider node disconnections and power consumption efficiency. Moreover, another roadmap would involve the inclusion of a static backbone, like in Wireless Mesh Networks (WMN), by reducing the mobility of some router nodes. In addition, some solutions have been proposed at application layer, from loss differentiation algorithms, which allow applications to adapt to the type of losses (congestion, route 
breakage, etc.), to new video coding techniques, such as Layered Coding (LC) [2] or Multiple Description Coding (MDC) [3]. MDC is a video coding technique capable of enhancing error resilience of a video delivery system by means of providing multiple video flows with redundant and enhancing information. This encoding technique is specially useful in multipoint-to-point transmissions due to the disjuntion of packet routes. This scheme makes packet losses more independent and increases the packet delivery probability. Among other solutions, MDC appears as one of the most suitable solution in multi-hop networks due to the possibility of using disjoint paths towards destination.

Another interesting mechanism used to minimize the burst effect and the unpredictability of packet losses is the Forward Error Correction (FEC) mechanism [4]. This technique consists of adding redundant data to the previously compressed signal, which allows the receiver to correct some errors without the need for any retransmission. However, this redundancy increases the total amount of bits required to transmit a specific content and, consequently, compression efficiency is reduced.

Besides the aforementioned proposals regarding videostreaming services over ad hoc networks, we consider also interesting to conduct a study that could be easily implemented and tested in a real environment later. In this sense, we have evaluated a platform for video distribution, which is based on a hybrid wired and wireless delivery network. This scenario facilitates the study of the improvements provided by using MDC and FEC techniques, taking advantage of the multipoint-to-point nature of the system. In order to evaluate these improvements, it is important to pay attention to Peak Signal to Noise Ratio (PSNR) and packet delivery ratio, which are parameters related to the objective quality of the reconstructed videos. In order to achieve a video assessment closer to real user experience, other metrics have been taken into account, such as the decodable frame rate and the interruptions suffered at video rendering, as well as subjective quality tests.

The rest of this paper is organized as follows. Section 2 reviews the related work regarding MDC and FEC techniques. In Section 3 we thoroughly describe the parameters used to evaluate the QoS and QoE. Section 4 explains the proposed scenario, the evaluation process and some considerations regarding this study. Section 5 shows the simulation results of the video evaluation. Finally, we present the conclusions and future work in Section 6.

\section{RELATED WORK}

As discussed in the previous section, a video streaming service entails many challenges in MANET networks [5]. In order to solve the problems regarding wireless links and node mobility, several mechanisms are nearly required to be used simultaneously. Moreover, taking into account the limited resources of nodes, these solutions should be easy and simple to implement. Among different proposed solutions, this paper focuses on those related to video coding techniques. In this sense, it is essential to use efficient video encoders, which are able to offer a low bit rate with acceptable quality. Furthermore, due to the frequent burst packet losses that video flows can suffer in MANETs, the transmission of the same stream through different disjoint paths decreases the packet loss probability and improves the fault tolerance. By using multiple paths with sufficient path diversity, the probability that a link failure affects different paths simultaneously is notably reduced. Some proposals involving routing protocols provide mechanisms to obtain this path diversity [6], [7], [8]. Another proposed mechanism to obtain path diversity consists of using multiple sources to send several streams to the same destination node, which is called multipoint-to-point (MP2P) transmission [9]. This existence of disjoint (or partially disjoint) paths can be used to send different substreams through different paths [10], [11] which is very common when using MDC. MDC is a coding technique that generates several bitstreams, which are called descriptions, from the same media source. Every description can be decoded independently, providing a useful reproduction of the original stream. In order to decode the video stream, any description can be used. Moreover, the descriptions contain complementary information in order to improve the quality of the decoded video when the amount of received descriptions increases. Different levels of reconstruction qualities can be obtained from different subsets of these descriptions. In contrast to layer coding [12], there is no hierarchy among the descriptions so each one may be independently decoded. The fact that each description is independent and equally important makes the use of MDC suitable on lossy systems where there is no packet delivery priority, like in ad hoc networks or the Internet.

Furthermore, MDC can be used in conjunction with other techniques in order to improve packet error resilience, like in [13] where authors combine MDC with FEC algorithms for voice communications. Real-time applications have strict time restrictions, i.e. if a packet (or a frame) is received after a certain deadline, it becomes useless and it is considered lost. This makes quite unfeasible the retransmission of lost packets in real-time video over MANETs, since retransmissions often involve an unacceptable delay. 
Because of this, the use of FEC in real-time video transmissions turns out appropriate. [14] describes a FEC mechanism based on Raptor codes and layer coding, where an unequal packet-loss protection (UPLP) scheme is used for protecting different layers with different importance. On the other hand, [15] introduces a scalar coefficient for rating a multi-path routing topology in order to decide on the amount of FEC protection.

As mentioned above, there are two key concepts regarding video quality evaluation: Quality of Service and Quality of Experience. QoS is defined as a set of attributes that can be used to define the network's capability to meet the requirements of users and applications. QoS covers the concepts, parameters and methods needed to manage the interactions between applications, typically running in end-user terminals and in network nodes managed by network operators. QoS parameters include bit rates, delay properties and packet loss rates. However, these metrics fail to capture the subjectiveness associated with human perception and understanding. A better measure of subjective video perception is QoE. Various definitions for QoE have been proposed in the literature. For instance, International Telecommunication Union (ITU) [16] describes the QoE as the overall acceptability of an application or service as it is perceived subjectively by the end-user. Grega et al. [17] defines QoE as a set of QoS perceived by a user and in [18], Kilkki states that QoE is related to all relevant aspects that define how satisfied a person is with a service. According to Corrie et al. [19], QoE is how the user feels about how an application or service was delivered, relative to their expectations and requirements [19][20]. Therefore, both kinds of metrics are necessary to carry out a complete analysis of the results.

\section{VIDEO QUALITY MEASUREMENT: QUALITY OF SERVICE AND QUALITY OF EXPERIENCE}

In order to measure and predict the actual performance of a streaming service, system designers commonly use some QoS metrics as performance parameters. Typical QoS metrics include network latency, jitter and packet loss. These parameters are usually used as measures for the network performance itself. However, the ultimate measurement of quality for services offered by a multimedia streaming system is the satisfaction of the final user and the video quality experienced. Good QoS at network level not always ensures a good user experience [20]. Below a brief overview of video quality assessment is outlined and the subjective test selected for this study is explained.

\subsection{Objective Assessment}

In order to assess video quality in a video distribution system, some evaluation parameters have to be defined. In this sense, Video Quality Experts Group (VQEG) [21] classifies the video quality model into three categories according to the availability of the reference video sequence: full-reference (FR) models, comparing with the original sequence when the original sequence is entirely available to compare with; reduced-reference (RR) models, where the only access to the reference sequence is a partial information related to the original video; and no-reference (NR) models, where no information can be used as a reference.

Currently, in a full-reference simulation setup, the most widely used objective video quality metrics are PSNR and Mean Squared Error (MSE) [22]. PSNR is the ratio between the maximum possible power of a signal and the power of corrupting noise that affects the fidelity of its representation (mathematically, PSNR is just a logarithmic representation of MSE). MSE and PSNR are widely used because they are simple to calculate and mathematically easy to deal with for optimization purposes as well as having clear physical meanings. Nevertheless, other QoS metrics have been developed in order to achieve more accurate results such as the Video Quality Metric (VQM) [23] or the Structural Similarity Index (SSIM) [24]. SSIM is a full-reference metric designed to improve the traditional PSNR and is based on frame-toframe measuring of three components (luminance similarity, contrast similarity and structural similarity) and combining them into a single value. On the other hand, VQM is an objective method to closely predict the subjective quality based on human eye perception and other subjectivity aspects.

Furthermore, the performance of the video transmission has been evaluated in terms of Packet Delivery Ratio (i.e. packet throughput). This measurement is defined as the percentage of video packets successfully delivered to the destination against the total amount of sent packets. Nevertheless, every packet correctly received may not be used to decode the video flow due to errors in the reception of previous frames (if predictive encoding is used). In this case, additional measurements such as Decodable Frame Rate $(\mathrm{Q})$ are needed. When receiving video, a frame is decodable if and when all fragmented packets of this frame and other packets that this frame depends on are completely received and are 
decodable. Thus, Q parameter is defined as the number of decodable frames over the total number of frames sent by a video source [25]. This parameter should be less or equal than the throughput.

In addition, video interruptions, which have been previously defined in [26], have been used as a performance metric in this study. An interruption is observed when one or more consecutive frames cannot be decoded due to the loss of some video packets. The nature of the human visual system makes it very difficult for a viewer to notice distortion if only a small amount of consecutive frames are lost. When the number of lost frames increases beyond a limit, the distortion can be noticed. The seriousness of an interruption depends on how long the interruption occurs. Interruptions can be classified according to their seriousness as minor and major interruptions. An interruption can be considered minor if it causes a lesser distortion whereas a major interruption distorts the received video or even stops it momentarily. Considering the effect on user perception, the threshold has been established to 1 second [26]. Thus, we consider a major interruption when it lasts more than 1 second. It is worth mentioning that the frequency the interruptions occur is another parameter to be considered as well. Although the interruptions are measured taking into account objective data, their impact is strongly related to the subjective quality.

\subsection{Subjective Assessment}

As mentioned before, image and video processing community has long been using MSE and PSNR as quality metrics. The popularity of these two metrics comes from the fast computation and the simplicity of the formulas to understand and implement. Despite its popularity, PSNR only has an approximate relationship with the video quality perceived by human observers, simply because it is based on a byteby-byte comparison of data without considering what they actually represent. Therefore, the perceived quality of images or videos with the same PSNR can actually be very different. Moreover, as the PSNR value for a single image may not correspond with the quality perceived by the viewer, the average PSNR while evaluating video sequences does not always correspond with the quality experienced. This is due to the fact that instant PSNR can present high peaks resulting in a high average value, whereas the user could experiment gaps or high degradation in the rest of the video sequence. Figure 1 shows an example comparing two video sequences specifically chosen to explain this behavior; sequence 1, which has an average PSNR of $22.2 \mathrm{~dB}$, and sequence 2, with an average PSNR of $21.3 \mathrm{~dB}$. Although sequence 1 presents a higher PSNR mean value, it is observed that video quality falls down during almost 6 seconds, while the quality of sequence 2 only varies in a short range. This is also stated in [27] where a subjective analysis of video transmission using scalable coding is mentioned. Figure 1 (right) shows a zoomed area where this effect is more clearly visible. Sequence 2 has intermittent losses per frame but it is enough to maintain a better quality than sequence 1 during nearly 6 seconds.
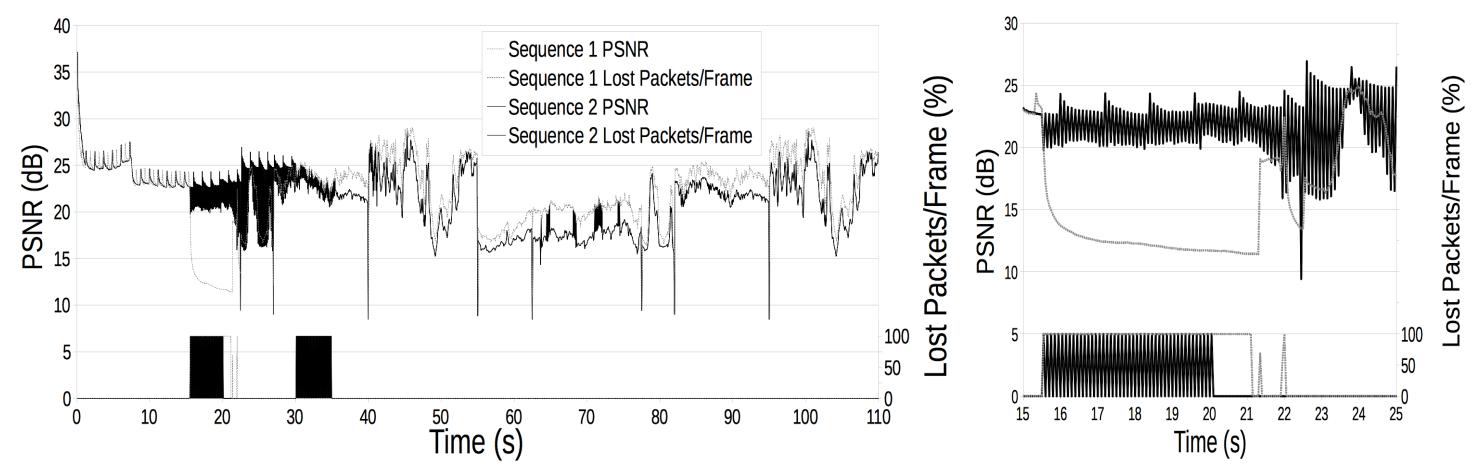

Figure 1 Example of PSNR and Lost Packets per Frame vs. Frame Number

Therefore, in order to complete the video quality evaluation, it is appropriate to conduct a subjective analysis. The subjective assessment consists in building a panel of human observers, which will evaluate sequences of video depending on their point of view and their perception. The test outputs can be obtained in terms of absolute scales or comparative scales. An example of an absolute qualitative scale is the Mean Opinion Score (MOS) scale, which was initially standardized by the ITU [28]. On the other hand, in comparative scales, observers set mutual relations between sequences.

There are some standard methods in the literature for conducting subjective video quality evaluations such as the ITU-R BT.500 [29]. This recommendation suggests mechanisms such as Single Stimulus (SS), Double Stimulus Impairment Scale (DSIS), Double Stimulus Continuous Quality Scale (DSCQS), Single Stimulus Continuous Quality Evaluation (SSCQE), Simultaneous Double Stimulus for Continuous Evaluation (SDSCE) and Stimulus Comparison Adjectival Categorical Judgment (SCACJ). All these 
variations are pretty much similar; differences are derived from the possibilities for constructing the subjective test methodology. The stimulus can be single, double or simultaneous double. The evaluation can be one step or continuous. The experiment may be single or may be repeated multiple times. The scale used for evaluation may differ in terms of continuity (continuous or non-continuous), direction (evaluation of quality or impairment) as well as relation (absolute or comparative). The ITU-R BT.500 gives examples of assessment problems and recommended methods to be used.

One of the most commonly method used is the SDSCE method in which an observer is presented with a pair of short video sequences 1 and 2, simultaneously. The observers are requested to check the differences between the two sequences and judge the fidelity of the impaired video compared with the reference by marking on a continuous scale according to Table 1 . The observers are aware of which sequence is the reference and which one is under test conditions. In this paper, this method is used with the aim of evaluating the improvements in QoE provided by MDC and FEC, as discussed in the results section.

Table 1. SDSCE test

\begin{tabular}{|c|l|}
\hline Value & \multicolumn{1}{|c|}{ Comparison } \\
\hline 3 & seq.2 much better than seq.1 \\
\hline 2 & seq.2 better than seq.1 \\
\hline 1 & seq.2 slightly better than seq.1 \\
\hline 0 & seq.2 the same as seq.1 \\
\hline-1 & seq.2 slightly worse than seq.1 \\
\hline-2 & seq.2 worse than seq.1 \\
\hline-3 & seq.2 much worse than seq.1 \\
\hline
\end{tabular}

\section{HYBRID ARCHITECTURE FOR VIDEO DISTRIBUTION}

In this section, it is described a study that is carried out in order to select the optimum number of descriptions and FEC parameters needed for the testbed. Hence, the impact of using different number of descriptions is analyzed, and FEC parameters such as the code rate or block size are evaluated with the aim of selecting the most appropriate values for the study. The proposed architecture for video distribution is also described below as well as the simulation environment.

\subsection{MDC and FEC Considerations}

There are several procedures for generating descriptions. Usually, a source video stream is divided into different groups or substreams. These substreams are encoded separately and used as individual descriptions in the system. Some other methods use interdependent descriptions with correlated information to improve compression efficiency [30]. Segmentation of video sequence can be carried out in temporal, spatial or frequency domain. In this paper, a temporal domain multidescription codec is implemented, based on a standard MPEG-4 codec. In particular, temporal domain multidescription is used in this paper due to the simplicity on the implementation of both encoder and decoder.

In order to illustrate the video reconstruction at reception, Figure 2 depicts how the decoding process would be in a real video sequence using SDC and temporal MDC with two descriptions when frame losses occur. From original raw video sequence, symbol $\mathrm{O}$ represents a frame correctly received and symbol X means that the frame is lost or not fully decoded. In SDC, the video flow is interrupted and luckily, most players might freeze or repeat the last decoded frame (otherwise, a black screen is displayed instead). On the other hand, when some frames from one description are lost, MDC replicates the last frame correctly received from the other description, resulting in a video stream with half frame rate but with neither interruptions nor artifacts and, therefore, the overall experienced quality is improved. 


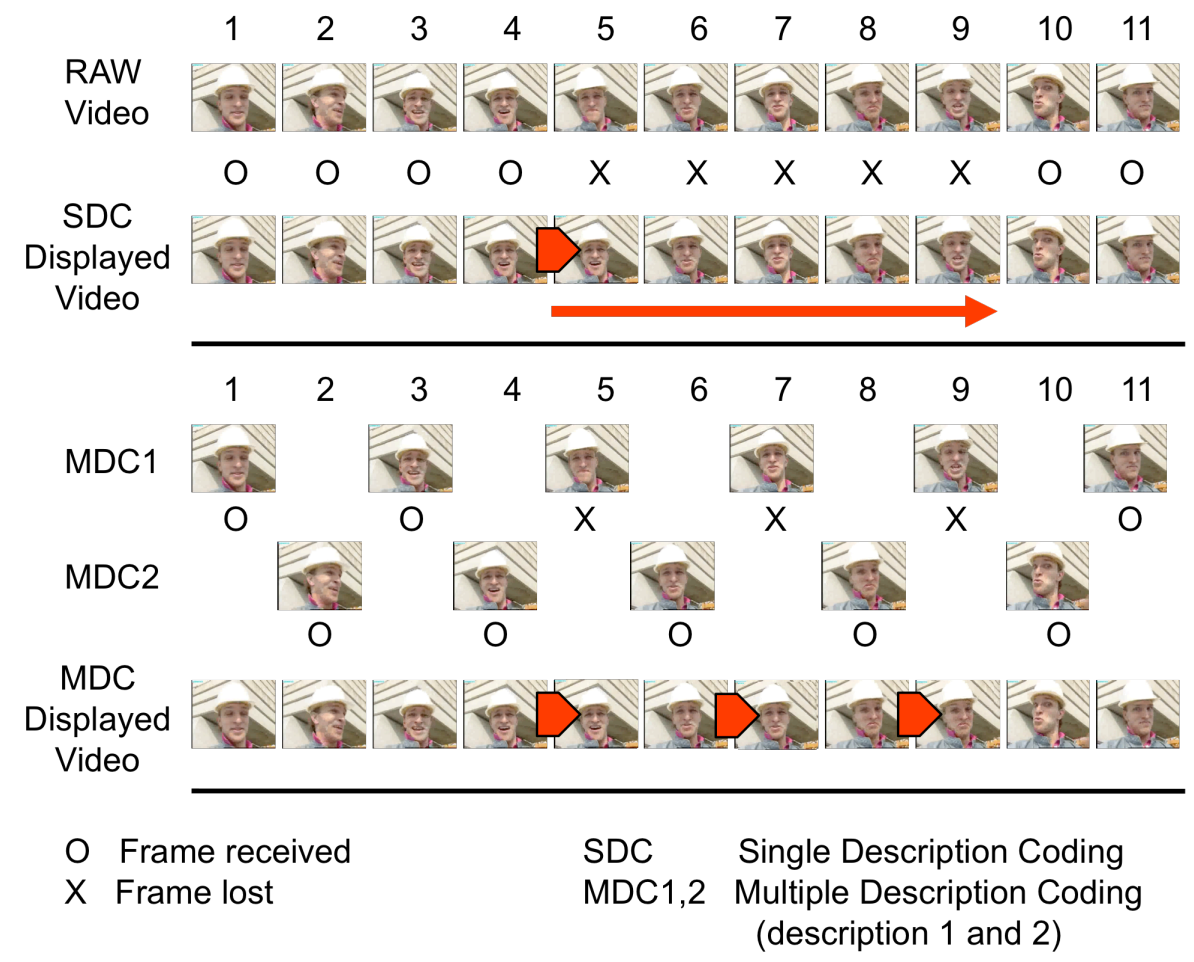

Figure 2. Decoding with SDC (up) and MDC (down) when frame losses occur

If losses occur in every description at the same time, no video reconstruction is obviously possible. Thus, the descriptions must be sent through disjoint paths to try to make losses independent in each description. In this paper, multiple sources have been used to send the descriptions to the same destination node. As shown below, the proposed architecture design is suitable for using this transmission mechanism.

It is worth noting that by encoding each substream independently, the overall required bandwidth for multidescription transmissions is greater than the one required for SDC. This drawback is due to the fact that the substreams are generated from non-consecutive frames causing lower compression efficiency. The more dynamic the video sequence, the bigger the mean frame size. Mean frame size is also increased when the amount of descriptions grows. Figure 3 illustrates the Cumulative Distribution Function (CDF) of the video frame size of a video sequence used in the simulations. It can be observed that as the number of descriptions increases, also does the frame size. For example, for a video encoded in a single description, there is a probability of $90 \%$ for the frame size to be lower than 5 KBytes (note that frame is later packetized for transmission). For two descriptions, this frame size represents approximately the $60 \%$, and for 3,4 and 5 descriptions, it is among $40 \%$ and $45 \%$. This shows a rather remarkable difference in the frame size, between encoding the video in two or three descriptions.

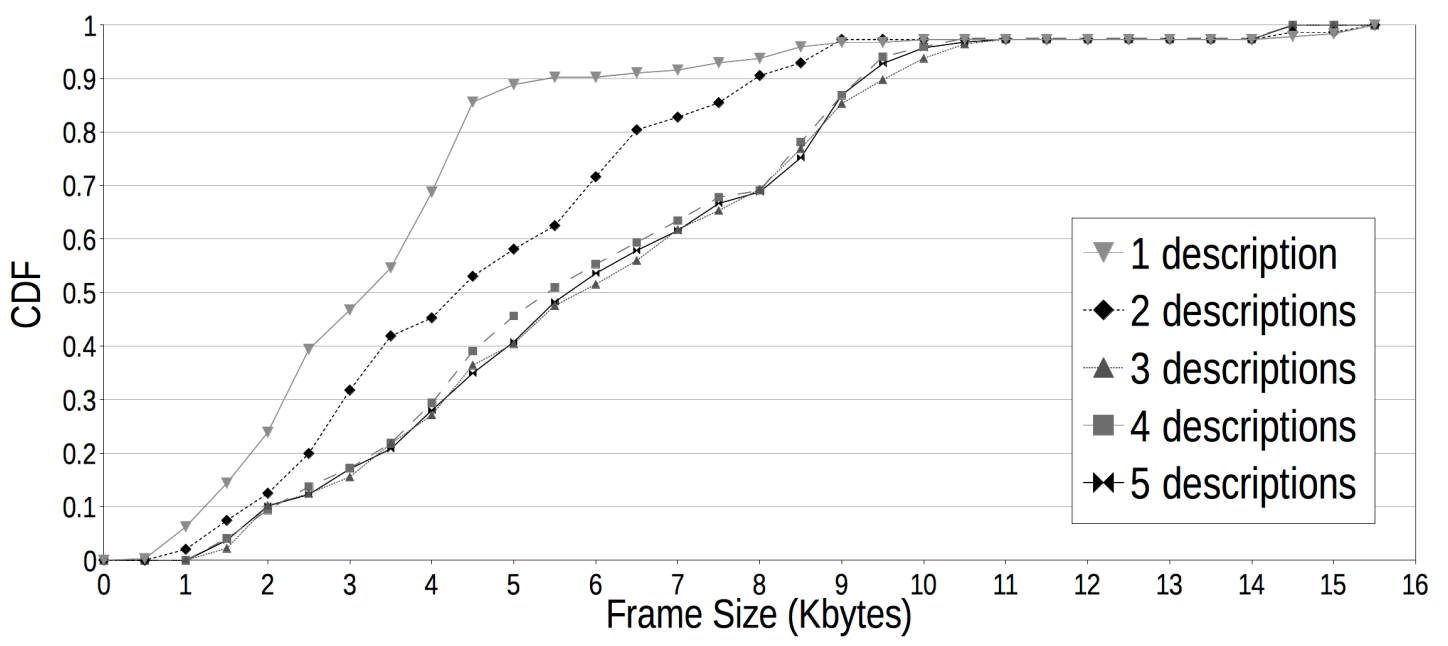

Figure 3. CDF of Video Frame Size 
As shown in Figure 3, the video frame size increases with the number of generated descriptions so the resulting frame size limits the amount of descriptions that can be used. In [26], this trade-off is studied and the authors conclude that the optimum values are 2 or 3 descriptions, depending on the available bandwidth and the desired quality of service. However, the use of 3 or more descriptions implies an increase of the system complexity while improvements in quality of service are not much significant. In addition, by increasing the frame size, the required bandwidth to transmit the video also increases and that increment depends on the type of video used, as previously explained. By comparing with the video sequence used as reference in this study, the average frame size increases by $16 \%$ when using two descriptions and increases by $31 \%$ when using three. Therefore, taking into account that the available bandwidth is a very limiting resource in mobile ad hoc networks, two descriptions are definitely used in this testbed.

Moreover, FEC also introduces traffic overhead, so this is another issue that must be taken into consideration. The degree of protection provided by FEC depends on the amount of redundancy added to the information to be transmitted. The higher the percentage of redundancy, the better the resistance against losses. However, this entails adding more traffic to the network. To analyze the impact of using FEC, a study has been carried out regarding the parameter values needed to encode FEC packets.

The FEC encoding scheme is based on block coding, i.e. data packets are grouped into blocks designated by $(n, k)$, where $k$ is the number of video packets to protect and $n$ is the total amount of packets generated. Therefore, $n-k$ packets are generates with FEC information from the $k$ video packets that form the block. These FEC packets must be generated from linearly independent combinations of data packets and, ideally, data packets can be fully recovered if and only if any $k$ packets from the block are received at least. In addition, as long as FEC operates in a block of packets, this block size directly affects the ability to correct mistakes related to burst losses, resulting in the loss of multiple consecutive packets. This is an important point because in ad hoc networks, losses often occur in bursts, so the FEC algorithm must be resistant to this kind of losses. It is worth mentioning that the FEC mechanism used in the simulation protects the video flow at packet level, not at frame level, so the FEC algorithm is applied to video stream after packetization. FEC packets are also packetized over a real-time transport protocol to be sent through network [31]. At the receiver, both FEC and original media packets are received. If no media packets are lost, FEC packets can be ignored. When losses occur, FEC packets can be combined with other received video packets to recover all missing data, as long as at least $k$ packets of the same block have been received. Otherwise, only those video packets that are correctly received are used to reconstruct the video. This is the behavior of an ideal FEC encoder and so is the mechanisms used in the simulations. As mentioned before, FEC code can be defined depending on the number of video packets that will constitute a block and the number of FEC packets (i.e. parity packets) that will be generated from each block. This relationship between data packets and the total amount of packets sent is the code rate. Therefore, different combinations of block size and code rate can be used. In this sense, two main factors have been taken into account when selecting the appropriate FEC parameters: the delay and the traffic overhead caused by FEC.

In ad hoc networks, packet losses often occur in bursts, so that for the same FEC level, better results are obtained (i.e. higher number of packets are recovered) by using a large block size. However, it is worth noting that the block size directly influences the video playing delay, since the receiver has to wait for a complete video block to rebuild and play the video. This also implies that the receiver must have a buffer with enough capacity to store all packets in the block, i.e. video packets plus FEC packets. Hence, rendering delay is directly related to the block size, because when some video packets are lost, the receiver has to wait for FEC packets, which are sent after the entire video block. Table 2 shows the delay introduced in reception depending on the block size. These values have been calculated from video trace files and represent the block delay depending on the block size selected. At reception, a buffer of 5 seconds is used as the play-out buffer in order to absorb the frame jitter, like in [26]. Taking this into account, a block size of 128 packets was selected, which produces an average delay of 3.8 seconds.

Table 2. Delay vs. Block size

\begin{tabular}{|c|c|c|c|c|}
\hline Block size (packets) & 32 & 64 & 128 & 256 \\
\hline Delay (s) & 0.9 & 1.9 & 3.8 & 7.2 \\
\hline
\end{tabular}

Regarding FEC overhead, it can be adjusted depending on the desired level of protection. This overhead is represented by $k / n$, which is the code rate. The higher the code rate the larger the burst of lost packets that can be recovered. It is worth noting that at higher node speeds, packet losses are likely to occur more frequently, so different code rates could be considerate depending on node mobility. In this sense, the 
burst size of lost packets has been measured and results are shown for speeds of $2.5,5$ and $7.5 \mathrm{~m} / \mathrm{s}$ on one hand, and 10, 12.5 and $15 \mathrm{~m} / \mathrm{s}$ on the other hand. The simulation scenario and a thoroughly analysis of the results are explained in further sections. Figure 4 shows the cumulative percentage of interruptions regarding the duration in seconds, for low and high speeds. As pointed in Figure $4 \mathrm{~b}$, almost $58 \%$ of interruptions could be avoided with a code rate of 2/3 (i.e. 64 FEC packets for each block of 128 data packets). For low speeds, Figure 4a shows that interruptions tend to be mainly shorter. Therefore, in order to reduce the FEC overhead and make more efficient use of bandwidth, 52 parity packets have been selected for simulations with low mobility, where more than $50 \%$ of interruptions can be still recovered. For static environments (i.e. $0 \mathrm{~m} / \mathrm{s}$ ) no FEC has been used in simulations because packet losses are almost inexistent and FEC would simply cause an inefficient use of bandwidth.

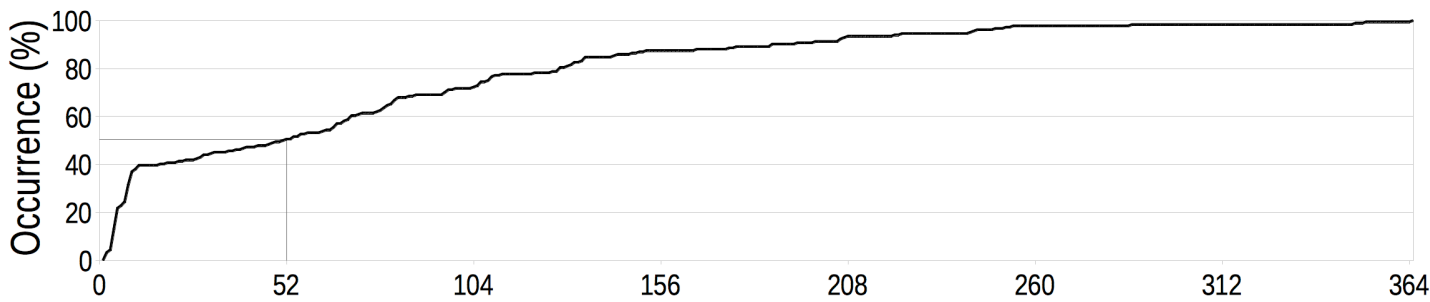

Burst Size (packets)

(a)

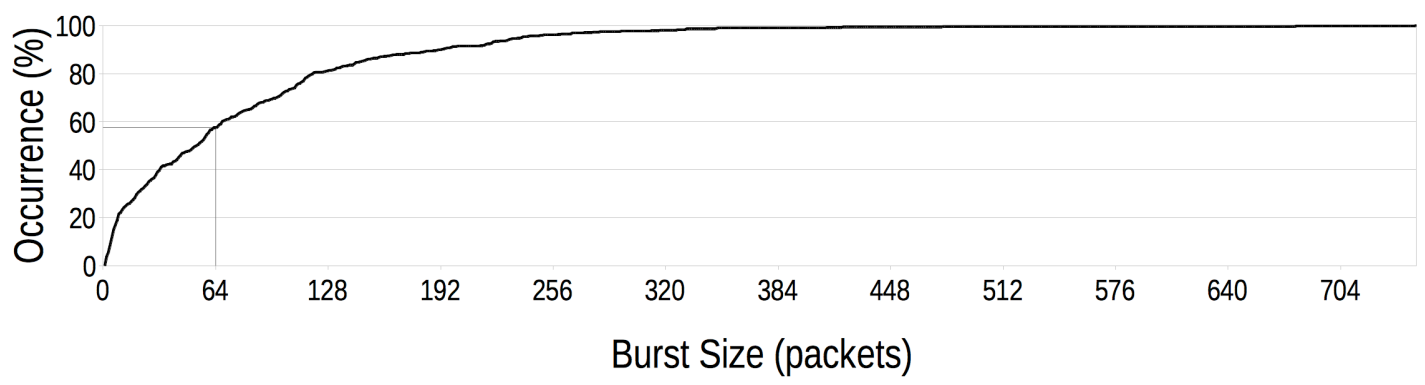

(b)

Figure 4. Cumulative Percentage of Interruptions Regarding its Duration for low speeds (a) and high speeds (b)

Regarding the costs of redundancy, Figure 5 depicts the extra delay and overhead caused by MDC, SDC + FEC and MDC + FEC compared with SDC. This bar graph shows the drawbacks of using any of these techniques regarding delay and traffic overhead. The results about subjective quality obtained in next Section can also be taken into account in conjunction with these in order to estimate the trade-off between quality and delay or overhead.

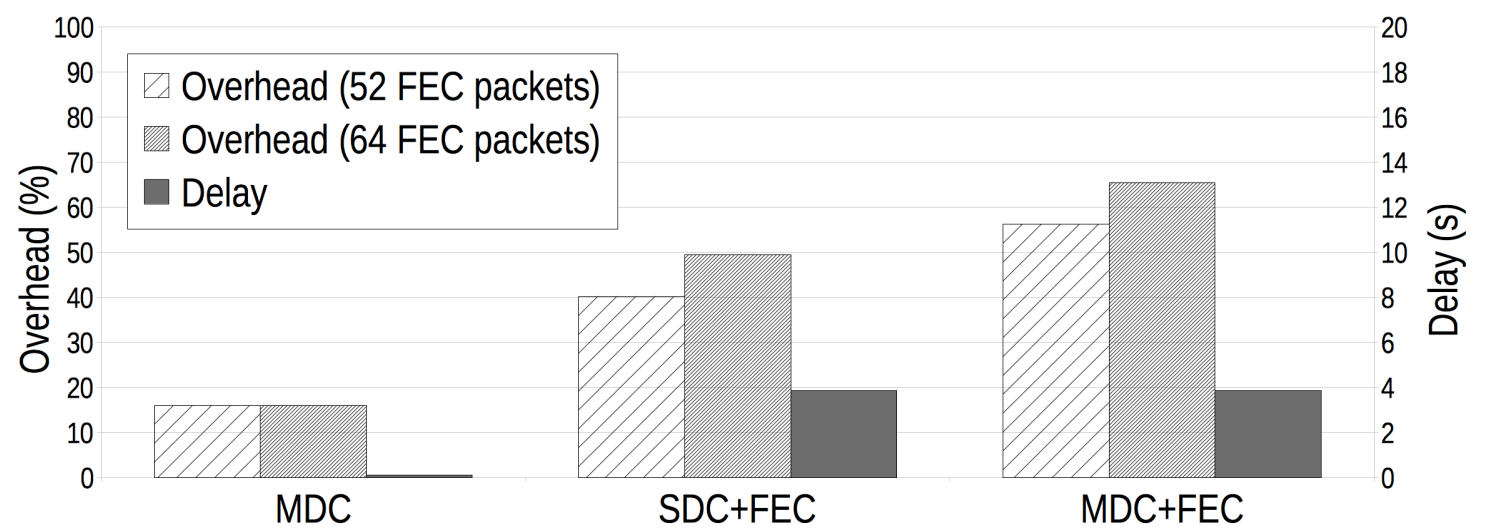

Figure 5. Overhead and Delay comparison in respect of SDC

\subsection{Proposed Scenario}


The proposed architecture for the content distribution system is based on a hybrid fixed and ad hoc network. Figure 6 shows the layout of the aforementioned scenario. It consists of a videostreaming server deployed in a wired network connected to a wireless ad hoc network through gateway devices. A group of mobile nodes form the wireless ad hoc network and act as hypothetical video clients. The ultimate goal of this work is to implement this platform on the campus. Each Wi-Fi access point is placed on both sides of the scenario to obtain disjoint paths to destination. Client nodes could be laptops or mobile phones that belong to students and staff, which can be moving around the campus. Simulations from this scenario can offer results about the video quality received on these devices under certain mobility conditions and FEC level.

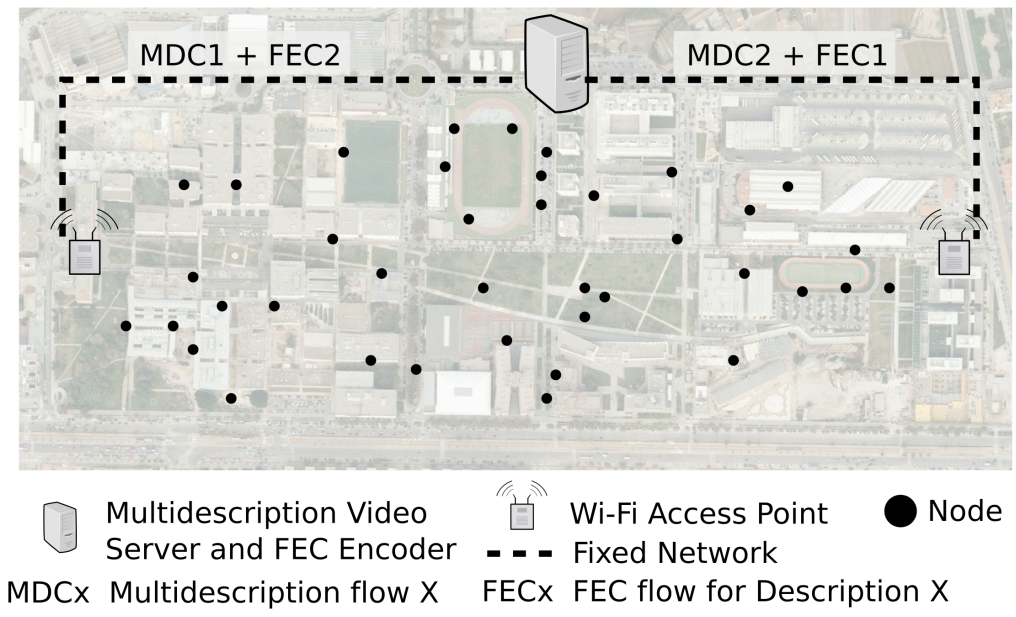

Figure 6. Hybrid fixed-ad hoc network scenario

The streaming service works as shown in Figure 7. A raw video in YUV format is encoded into two descriptions. This is achieved by splitting the original video frames in subsets depending on their time position. Therefore, the raw video is separated into two descriptions with a frame rate half of the original frame rate. So, the video content is encoded in several descriptions so they can be sent separately. The streaming server sends video description and FEC flows to the node that requests them. Access nodes are ad hoc nodes connected to the content server through a wired network. These nodes are placed along the scenario and are also part of the ad hoc network. Unlike the other nodes, access nodes have two interfaces in order to retransmit packets from the server in the wired network to the wireless nodes in the ad hoc network. Wireless nodes arbitrarily move within a delimited coverage zone. Any node in the network can request a video from the server so the routing protocol has to ensure that video and FEC packets are delivered to the destination node. Devices not involved in any communication can act as routers for the traffic of other connections. The routing protocol used was OLSR (Optimized Link State Routing) [32]. OLSR is a proactive routing protocol, i.e. nodes maintain routing tables by sending periodic messages. These messages cause some overhead in the network but, on the other hand, any node is able to establish a route to the destination at any time without further delay (if network topology is rather stable). 


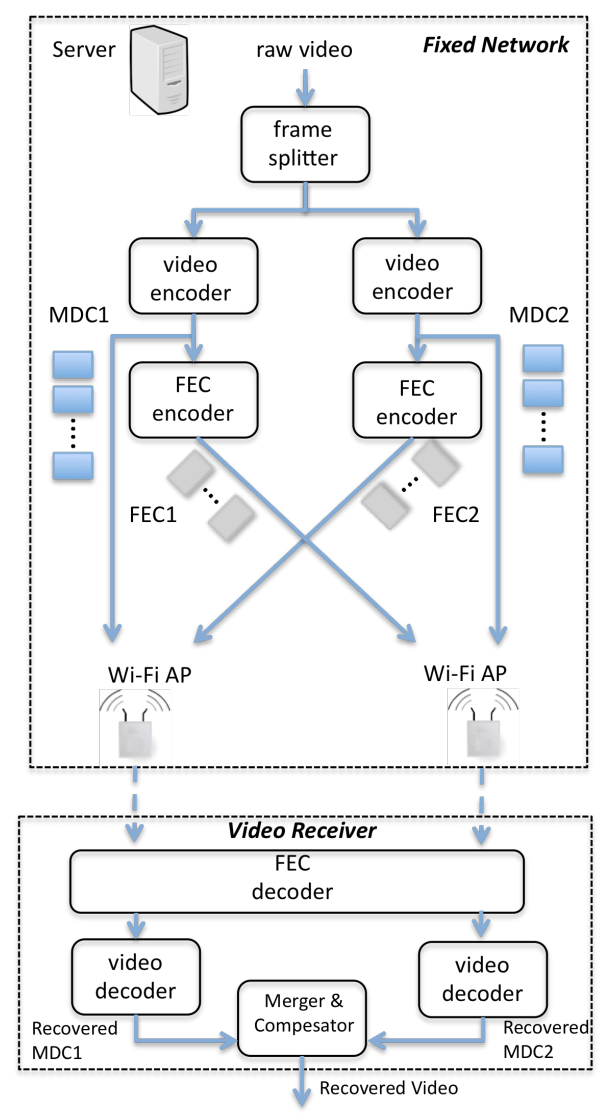

Figure 7. Streaming service workflow

When any device requests a video stream, the content server transmits the video descriptions and FEC flows through the access points. Each description is sent and transmitted through a different access point using static routing configuration. Additionally, the corresponding FEC flow for each description is transmitted through the opposite access point, i.e. description and FEC are also sent through disjoint paths. When video and FEC packets arrive to the access point, it retransmits them to the wireless network. If the requesting node is not in range, intermediate nodes behave as routers. Access points are placed at opposite edges in the scenario so descriptions and FEC flows can be transmitted over likely disjoint paths and no routing protocol modification is needed. As a good consequence of using MDC, mobile devices with limited processing capabilities could request only one description in order to play the stream at a lower rate. Moreover, devices with constrained bandwidth could also avoid congestion with this method.

Regarding the simulation environment, the simulations were carried out under the well-known network simulator NS-2 and the Evalvid tool [33] for video tracing and evaluation purposes. The simulation environment consists of 50 wireless nodes random scattered over an area of $1200 \times 600 \mathrm{~m}$. Two descriptions with their respective FEC flows are transmitted from different nodes towards the same receptor performing a multipoint-to-point transmission. These nodes are used to simulate the distributed access points that allow connecting the multimedia server to the ad hoc network. The radio model is based on the Two-Ray Ground Propagation Model and the standard 802.11b at 11 Mbps. Node transmission range is approximately $250 \mathrm{~m}$. Each node moves according to the Random Waypoint (RWP) model, i.e. the wireless node randomly selects a destination, then it moves in the direction of this location at a certain speed and, when it arrives to destination, it pauses during the interval known as pause time. With the aim of evaluating the influence of node movement on the quality of video transmission, a number of random scenarios were simulated where each node moves using the RWP model at $0 \mathrm{~m} / \mathrm{s}$ (all nodes are static), $2.5 \mathrm{~m} / \mathrm{s}, 5 \mathrm{~m} / \mathrm{s}, 7.5 \mathrm{~m} / \mathrm{s}, 10 \mathrm{~m} / \mathrm{s}, 12.5 \mathrm{~m} / \mathrm{s}$ and $15 \mathrm{~m} / \mathrm{s}$, as maximum speed. The results are obtained with a confidence level of $95 \%$.

The reference video stream has been created by combining some well-known test sequences such as bus, mobile, stefan, tempete and foreman, for the sake of diversity. The resulting video has 2200 frames and it has encoded at $20 \mathrm{fps}$ with a resolution of $352 \times 288$ pixels (CIF). Regarding MDC, two descriptions are generated by splitting the source in temporal domain. The original video and the descriptions are encoded 
in MPEG-4 with the GoP pattern IPPPPPPPPPPP. The reference video source is used as the SDC stream and it is encoded at an average bitrate below $200 \mathrm{kbps}$. This bitrate was deliberately selected as a tradeoff between quality and network bandwidth. The effect of intraflow contention in multihop paths over wireless networks [34] has been taken into account, which reduces the maximum bitrate that a flow can reach. Therefore, the mean PSNR of the encoded video, which has been used as reference video, is 25.66 $\mathrm{dB}$, in contrast with the higher quality that MPEG-4 codec can achieve. The video server generates FEC packets to protect the SDC stream and both of the MDC descriptions. Background traffic consists of 5 UDP connections with Constant Bit Rate (CBR) at $1 \mathrm{~KB} / \mathrm{s}$ between arbitrary nodes, which simulates low dense traffic. The simulation parameters are summarized in Table 3.

Table 3. Simulation parameters

\begin{tabular}{|l|l|}
\hline \multicolumn{1}{|c|}{ Parameter } & \multicolumn{1}{c|}{ Value } \\
\hline Terrain Area & $1200 \mathrm{~m} \times 600 \mathrm{~m}$ \\
\hline Transmission Range & $250 \mathrm{~m}$ \\
\hline Channel Capacity & $11 \mathrm{Mbps}$ \\
\hline Propagation Model & Two-Ray Ground \\
\hline Number of Nodes & 50 \\
\hline Mobility Model & Random Waypoint (RWP) \\
\hline Simulation Duration & 200 seconds \\
\hline MAC protocol & $802.11 \mathrm{~b}$ DCF \\
\hline Routing Protocol & OLSR \\
\hline Video Resolution & $352 \times 288(\mathrm{CIF})$ \\
\hline Background Traffic & $5 \mathrm{CBR} \times 1 \mathrm{~KB} / \mathrm{s}$ \\
\hline
\end{tabular}

\section{EVALUATION AND RESULTS}

The proposed hybrid architecture has been evaluated in a simulation environment to assess the performance of the video service using MDC and FEC. In this sense, the simulation process carried out could be summarized as follows. Firstly, the MDC codec performs a frame separation of the raw video in order to generate the descriptions. Every description is then independently encoded in MPEG-4 (note that any standard video codec could be used by following this scheme). Video and FEC traces are generated for each description from the video sequence and serve as traffic pattern for the video and FEC sources in NS-2. During the simulation, the source and destination nodes involved in the video transmission save information regarding the video and FEC packets sent and received, and their time stamps, into trace files. FEC traces are used to recover some video packets and generate new video trace files. Then, the received descriptions can be reconstructed from the generated traces and merged into a new video with the same temporal resolution and frame number as the original. As a consequence of the dynamic scenario, the resulting video sequence could be distorted due to possible losses. The results of the video evaluation using SDC, MDC and FEC are shown below.

Figure 8 shows the average PSNR value related to node speed. Both SDC and MDC are compared. The improvement of using FEC on each coding technique is also depicted. In general, the average PSNR diminishes when node speed increases, as can be expected because of rerouting and packet loss. At low speeds, SDC presents a better performance regarding PSNR (approximately $0.5 \mathrm{~dB}$ ). This is due to the fact that the temporal correlation between consecutive frames is higher in SDC. However, at higher speeds, MDC achieves better results (up to $3 \mathrm{~dB}$ ) since multidescription technique increases the probability of receiving at least one of the descriptions. Additionally, it can be observed that the fact of using FEC as error correction technique obtains a slight improvement in the average PSNR value. 


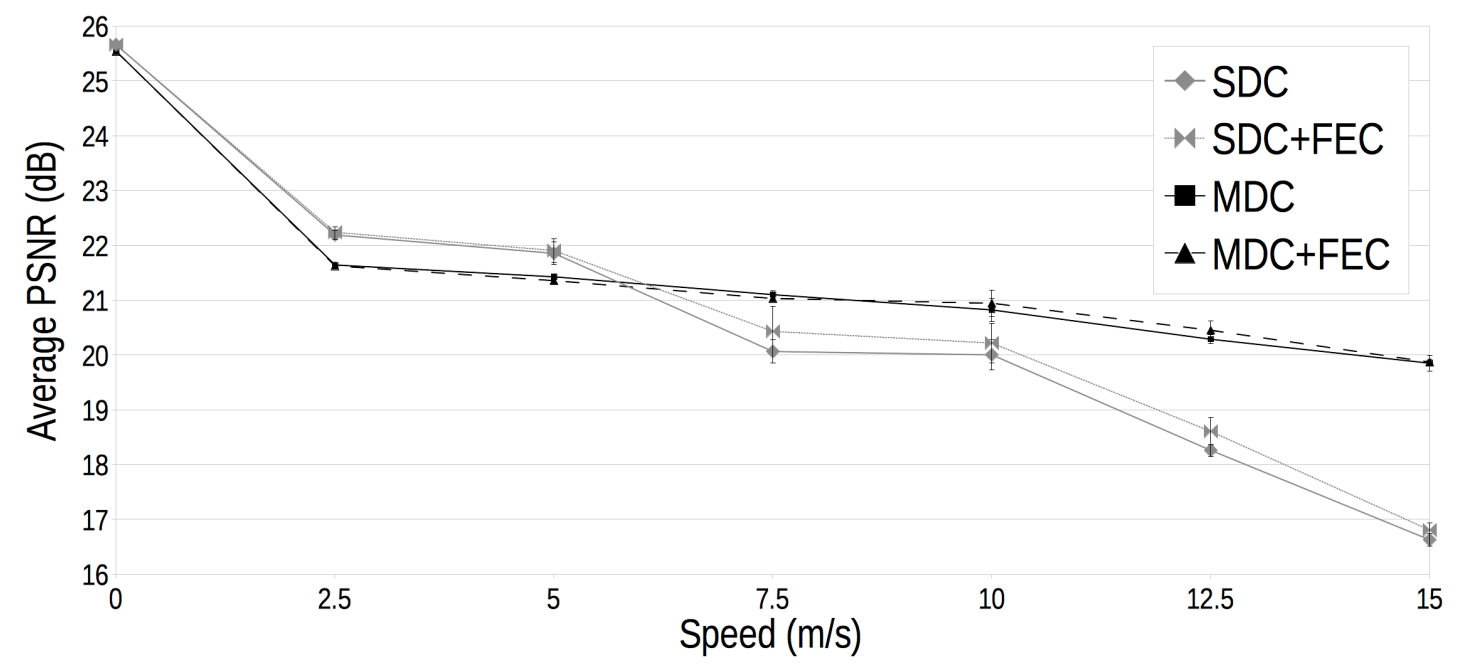

Figure 8. Average PSNR vs. Speed

As mentioned before, the average PSNR does not always correspond with the quality experienced by the user. Figure 9 illustrates the throughput and the decodable frame rate measured in the simulations. Results show that the percentage of correctly received frames decreases at higher node speeds. For medium and high speed, SDC suffers a greater amount of packet loss than MDC, accordingly with the previous PSNR results.

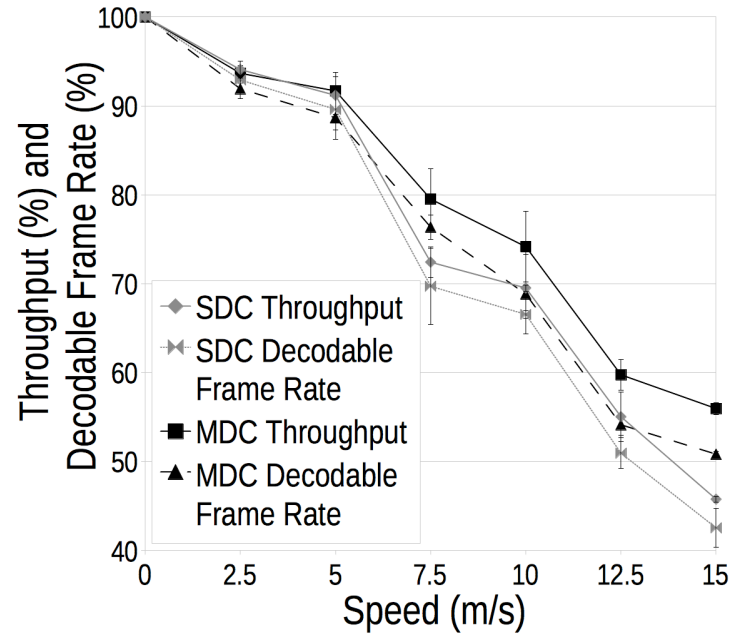

(a)

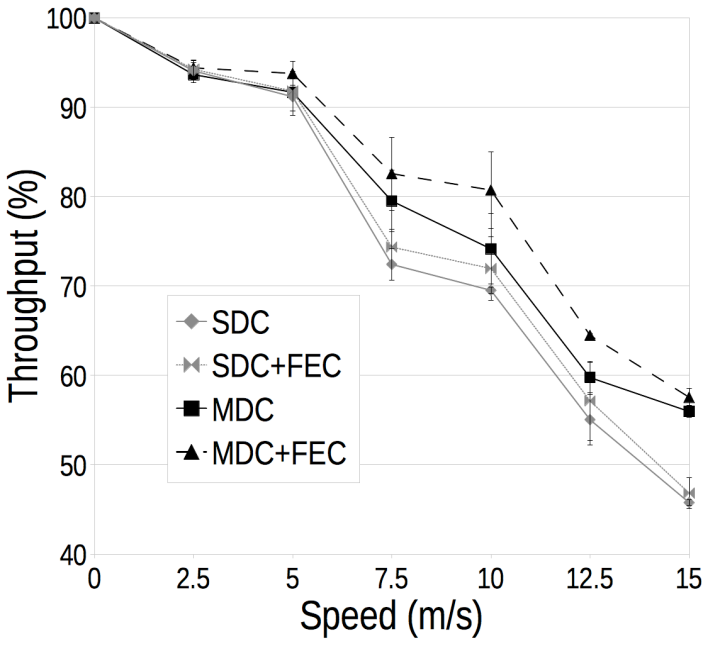

(b)

Figure 9. Throughput and Decodable Frame Rate vs. Speed (a) and Throughput vs. Speed (b)

As observed in Figure 9a, decodable frame rate is always below the throughput value. This occurs because not all the received packets can be used when reconstructing the video, due to the hierarchical and interdependent nature of MPEG-4 encoding. Q provides a measure of quality closer to that perceived by the user because it takes into account only the packets that are actually used when playing the video.

Furthermore, if the values for $2.5 \mathrm{~m} / \mathrm{s}$ and $5 \mathrm{~m} / \mathrm{s}$ are observed, both SDC and MDC have similar throughput, but the former obtains higher decodable frame rates at low speeds, so PSNR becomes higher. The difference in decodable frame rate is due to the fact that MDC affects the GoP structure of the reconstructed video. Therefore, if one consecutive frame of each description is lost inside the same GoP, the rest of the frames will not be fully decodable until next I frame, which takes longer than in SDC.

On the other hand, some additional lost packets can be recovered when using FEC. This can be seen in Figure 9b, as the throughput increase suggests. This throughput increase is most remarkable in MDC, probably because the FEC packets are sent through a different path than the one used to send the video. 
As expected, the path diversity reduces the probability for video packets to be lost together with the FEC information, allowing in this way to recover a higher percentage of lost packets.

Figure 10 and Figure 11 show the number and the aggregate length of minor and major interruptions respectively.
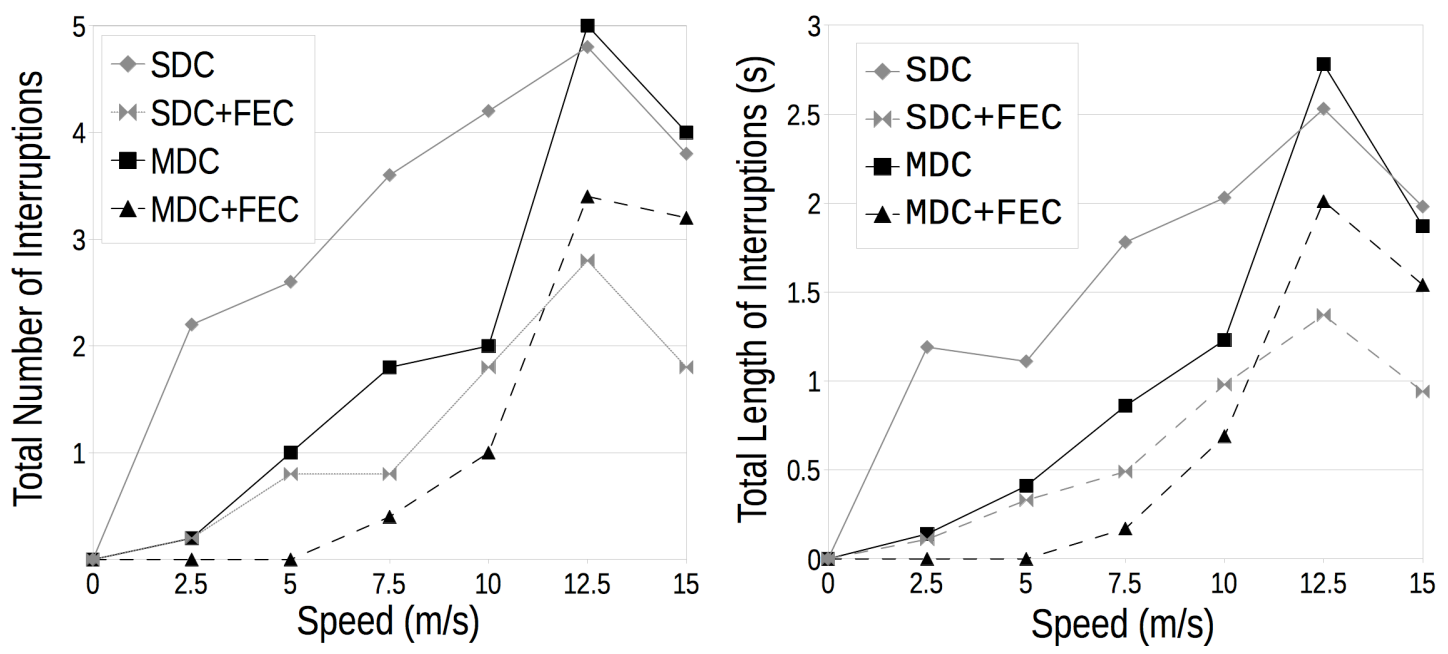

Figure 10. Number and Aggregate Length of Minor Interruptions vs. Speed

In Figure 10, MDC shows a better performance except at high speeds, where both mechanisms present similar results. The minor interruption decrease at $15 \mathrm{~m} / \mathrm{s}$ matches the increment of major interruptions. Likewise, it is observed that the number and duration of minor interruptions is reduced while using FEC. In this way, although PSNR does not show a significant increase with the use of FEC, by reducing interruptions subjective quality does actually improve.
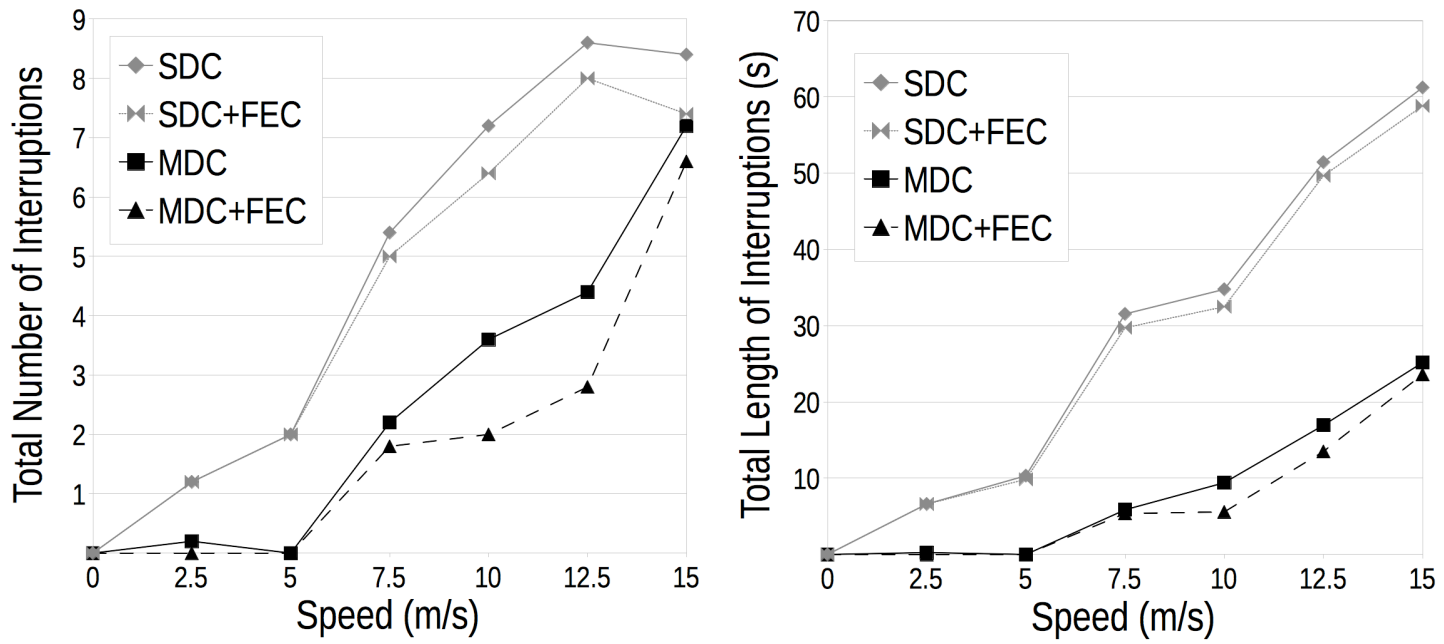

Figure 11. Number and Aggregate Length of Major Interruptions vs. Speed

Figure 11 clearly shows that using MDC noticeably reduces the number and the length of major interruptions. This is an important point because MDC is capable of providing video with an acceptable quality minimizing the pauses or video gaps caused by losses. This is due to the fact that the destination node probably receives information from at least one of the source descriptions.

Moreover, the use of FEC also allows reducing the number and duration of the major interruptions. As with the throughput and the decoding frame rate a more significant improvement is observed in MDC coding. This reveals that the use of different paths offered by the MDC scheme not only benefits the multidescription itself, but also increases the efficiency of error correction.

Finally, several subjective quality tests have been carried out to really assess and measure the degree of improvement in the video quality using MDC and FEC techniques. Figure 12 shows the results of the tests. The tests are performed using the SDSCE method, which has been previously described. Encoded 
videos using SDC are used as reference (seq.1 in Table 1) and they are compared with videos encoded in $\mathrm{MDC}$ and MDC + FEC (seq.2 in Table 1).

It can be observed that the obtained values are always positive, which means that for all speeds, users have experienced a better video quality using MDC compared with SDC. Furthermore, an increase in video quality is perceived when using FEC. This is mainly due to the fact that the use of FEC allows reducing the number and duration of interruptions, corroborating the results shown above.

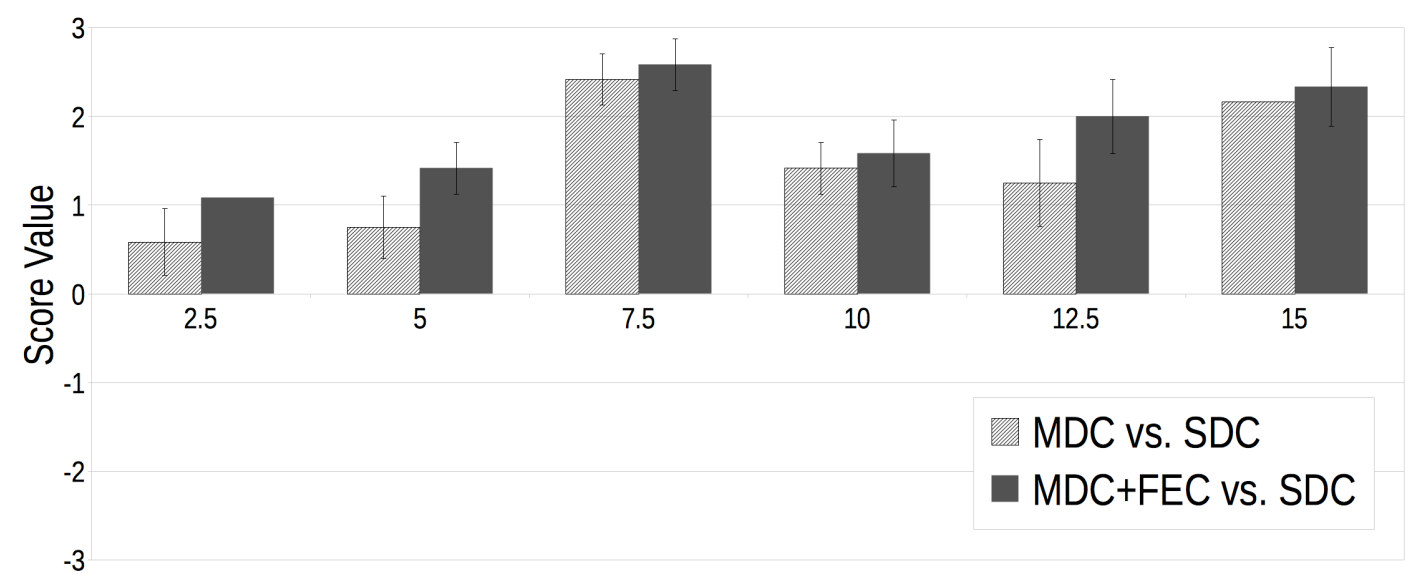

$$
\text { Speed }(\mathrm{m} / \mathrm{s})
$$

Figure 12. Results of the SDSCE test comparing MDC vs. SDC and MDC+FEC vs. SDC

From the results of the SDSCE test it can be concluded that the MDC allows improving the QoE in realtime video transmissions over mobile ad hoc networks, and the degree of improvement is more clearly visible at high speeds, when high packet losses take place. Moreover, the FEC scheme used allows improving the QoE, especially at low speeds, even if these results are not reflected in the average PSNR value. However, when node mobility increases, video interruptions are much longer and cannot be recovered with FEC. Ultimately, the number of packets that can be recovered is not directly proportional to the improvement in PSNR and subjective quality. By recovering only a few packets, the human eye may not perceive any quality improvement since a whole frame might not be fully recovered. In addition, given the hierarchical structure of MPEG-4, the type of the recovered frame also has a high influence on the results.

\section{CONCLUSION}

In this paper, we have performed a study considering a scenario for video distribution in a hybrid wired and wireless ad hoc environment with mobile nodes. In such a dynamic scenario, quality of video transmissions needs to be improved by using error resilience mechanisms.

For this purpose, we have studied the Multiple Description Coding and Forward Error Correction, and compared these with traditional single-flow video coding mechanism. Results have shown that MDC noticeably increases PNSR for high speeds (up to $3 \mathrm{~dB}$ ) and reduces the number and length of interruptions, providing a video with acceptable quality along the entire transmission. This is achieved because of using disjoint paths in a multipoint-to-point architecture and FEC mechanism, which has demonstrated to decrease the number and length of video interruptions. This reduction of video stream interruptions and the acceptable mean quality achieved allow users to obtain a better QoE, as is confirmed by the positive values of the SDSCE test. These results facilitate the system implementation on a real environment using the parameters obtained from simulations. However, PSNR and throughput results show a mediocre performance when node mobility is high (more than $10 \mathrm{~m} / \mathrm{s}$ ).

With the aim of improving the overall system performance, we plan to evaluate MDC and FEC techniques together with hierarchical routing in WMN (with a static wireless backbone) and use adaptive algorithms to select different video sources and change between FEC code rates in transmission time.

\section{ACKNOWLEDGMENT}


This work was supported by project MIQUEL (TEC2007-68119-C02-01/TCM) of the Spanish Ministry of Education and Science. The authors would like to thank the Editor and the reviewers for helpful suggestions to improve the quality of this paper.

\section{REFERENCES}

[1] Boukerche A (2009) Algorithms and Protocols for Wireless and Mobile Ad Hoc Networks. John Wiley \& Sons Inc.

[2] Schwarz H, Marpe D, Wiegand T (2007) Overview of the Scalable Video Coding Extension of the H.264/AVC Standard. IEEE Transactions on Circuits and Systems for Video Technology 17:11031120 .

[3] Apostolopoulos JG, Wong T, Tan W, Wee SJ (2002) On Multiple Description Streaming with Content Delivery Networks. IEEE INFOCOM

[4] Xunqi Y, Modestino JW, Bajic IV (2005) Performance analysis of the efficacy of packet-level FEC in improving video transport over networks. IEEE International Conference on Image Processing 2:177-180.

[5] Lindeberg M, Kristiansen S, Plagemann T, Goebel V (2011) Challenges and techniques for video streaming over mobile ad hoc networks. Multimedia Systems, 17:51-82.

[6] Wei W, Zakhor A (2004) Robust multipath source routing protocol (RMPSR) for video communication over wireless ad hoc net- works. Proceedings of IEEE International Conference on Multimedia and Expo 2:1379-1382.

[7] Hsieh MY, Huang YM, Chian TC (2007) Transmission of layered video streaming via multi-path on ad hoc networks, Multimedia Tools and Applications 34:155-177.

[8] Gharavi H (2008) Multi-channel for multihop communication links. International Conference on Telecommunications, $1-6$.

[9] Keisuke U, Cheeonn C, Hiroshi I (2008) A Study on Video Performance of Multipoint-to-Point Video Streaming with Multiple Description Coding Over Ad Hoc Networks. EEJ Transactions on Electronics, Information and Systems, 128:1431-1437.

[10] Rong B, Qian Y, Lu K, Hu RQ, Kadoch M (2010) Multipath Routing over Wireless Mesh Networks for Multiple Description Video Transmission. IEEE Journal on Selected Areas in Communications 28:321-331.

[11] Liao Y, Gibson JD (2011) Routing-Aware Multiple Description Video Coding Over Mobile Ad-Hoc Networks. IEEE Transactions on Multimedia, 13:132-142.

[12] Schierl T, Stockhammer T, Wiegand T (2007) Mobile video transmission using scalable video coding. IEEE Trans. Circuits Syst. Video Technol. 17:1204-1217.

[13] Gandikota VR, Tamma BR, Murthy CSR (2008) Adaptive-FEC Based Packet Loss Resilience Scheme for Supporting Voice Communication over Adhoc Wireless Networks. IEEE Transactions on Mobile Computing 7:1184-1199.

[14] Schierl T, Ganger K, Hellge C, Wiegand T, Stockhammer T (2006) SVC-based multisource streaming for robust video trans- mission in mobile ad hoc networks. IEEE Wireless Communication 13:96-103.

[15] Gabrielyan E, Hersch R (2006) Reliable multi-path routing schemes for real-time streaming. International Conference on Digital Telecommunications 65-65.

[16] ITU - International Telecommunication Union. "Definition of Quality of Experience (QoE)", Reference: TD 109rev2 (PLEN/12), 2007.

[17] Grega M, Janowski L, Leszczuk M, Romaniak P, Papir Z (2008) Quality of Experience Evaluation for Multimedia Services. Przegląd Telekomunikacyjny i Wiadomości Telekomunikacyjne 4:142153.

[18] Kilkki K (2008) Quality of Experience in Communications Ecosystem. Journal of Universal Computer Science 14:615-624.

[19] Corrie B et al. (2003) Towards Quality of Experience in Advanced Collaborative Environments. Third Annual Workshop on Advanced Collaborative Environments. 
[20] Ni P (2009) Towards Optimal Quality of Experience Via Scalable Video Coding. Mälardalen University Press Licentiate Theses, Sweden.

[21] VQEG (2008) “Video quality experts group". Available online: http://www.vqeg.org.

[22] Winkler S, Mohandas P (2008) The Evolution of Video Quality Measurement: From PSNR to Hybrid Metrics. IEEE Transactions on Broadcasting 54:660 - 668.

[23] Pinson MH, Wolf S (2004) A new standardized method for objectively measuring video quality. IEEE Transactions on Broadcasting 50:312 - 322.

[24] Wang Z et al. (2004) Image Quality Assessment: From Error Visibility to Structural Similarity. IEEE Transactions on Image Processing 13:600-612.

[25] Kao KL, Ke ChH, Shieh CH (2006) An Advanced Simulation Tool-set for Video Transmission Performance Evaluation, IEEE Region 10 Conference, 1-40.

[26] Chow CO, Ishii H (2007) Enhancing Real-time Video Streaming over Mobile Ad Hoc Networks using Multipoint-to-point Communication. Computer Communications 30:1754-1764.

[27] Zink M, Schmitt J, Steinmetz R (2005) Layer-Encoded Video in Scalable Adaptive Streaming. IEEE Transactions on Multimedia 7:75-84.

[28] ITU-T Recommendation P.910 (2000) "Subjective Video Quality Assessment Methods for Multimedia Applications", International Telecommunication Union, Geneva, Switzerland.

[29] ITU-R Recommendation BT.500-12 (2009) "Methodology for the Subjective Assessment of the Quality of Television Pictures", International Telecommunication Union, Geneva, Switzerland.

[30] Mao S et al. (2003) Video transport over ad hoc networks: multistream coding with multipath transport". IEEE Journal on Selected Areas in Communications 21:1721-1737.

[31] Li A (2007) RTP Payload Format for Generic Forward Error Correction. RFC 5109, Dec. 2007.

[32] Clausen T, Jacquet P (2003) Optimized Link State Routing Protocol (OLSR), RFC 3626.

[33] Ke CH et al. (2006) A novel Realistic Simulation Tool for Video Transmission over Wireless Network. Proceedings of the IEEE International Conference on Sensor Networks, Ubiquitous, and Trsutworthy Computing.

[34] Li J, Blake C, Couto DD, Lee H, Morris R (2001) "Capacity of ad hoc wireless networks" 7th Annual International Conference on Mobile Computing and Networking 16-21. 